\title{
Pharmacokinetics and Pharmacodynamics of High-Dose Piperacillin-Tazobactam in Obese Patients
}

\author{
John J. Veillette ${ }^{1} \cdot$ S. Alexander Winans ${ }^{1} \cdot$ Victoria K. Maskiewicz $^{2} \cdot$ James Truong $^{1} \cdot$ Ronald N. Jones $^{3}$. \\ Steven C. Forland ${ }^{1,4,5}$
}

Accepted: 12 February 2021 / Published online: 20 March 2021

(c) The Author(s) 2021

\begin{abstract}
Background and Objective Standard piperacillin-tazobactam (P-T) dosing may be suboptimal in obesity, but high-dose regimens have not been studied. We prospectively evaluated the pharmacokinetics and pharmacodynamics of standard-and high-dose P-T in obese adult inpatients.

Methods Those receiving standard-dose P-T with BMI $\geq 30 \mathrm{~kg} / \mathrm{m}^{2}$ weighing $105-139 \mathrm{~kg}$ or $\geq 140 \mathrm{~kg}$ were given up to $6.75 \mathrm{~g}$ or $9 \mathrm{~g}$ every $6 \mathrm{~h}$, respectively. Patients were monitored closely for safety. Elimination phase blood samples were drawn for 28 patients on standard and high doses to calculate the pharmacokinetic values using a one-compartment model. The likelihood of pharmacodynamic target attainment $(100 \% f \mathrm{~T}>16 / 4 \mathrm{mg} / \mathrm{L})$ on various P-T regimens was calculated using each patient's own pharmacokinetic values.

Results Piperacillin and tazobactam half-lives ranged from $0.5-10.6$ to $0.9-15.0 \mathrm{~h}$, while volumes of distribution ranged from 13.6-54.8 to 11.5-60.1 L, respectively. Predicted dose requirements for target attainment ranged from $2.25 \mathrm{~g}$ every $6 \mathrm{~h}$ in hemodialysis patients to a $27 \mathrm{~g} / 24$-h continuous infusion in a patient with a short P-T half-life. An amount of $4.5 \mathrm{~g}$ every $6 \mathrm{~h}$ would have met the target for only $1 / 12(8 \%)$ patients with creatinine clearance $\geq 80 \mathrm{~mL} / \mathrm{min}$ and $13 / 28(46 \%)$ for all enrolled patients. One patient (3\%) experienced an adverse event deemed probably related to high-dose P-T.

Conclusion Some patients required high P-T doses for target attainment, but dosing requirements were highly variable. Doses up to $6.75 \mathrm{~g}$ or $9 \mathrm{~g}$ every $6 \mathrm{~h}$ may be tolerable; however, studies are needed to see if high dosing, prolonged infusions, or real-time therapeutic drug monitoring improves outcomes in obese patients.
\end{abstract}

Clinical trial registration (clinicaltrials.gov) NCT01923363.

\section{Introduction}

Obesity, defined by the World Health Organization as a body mass index (BMI) $\geq 30 \mathrm{~kg} / \mathrm{m}^{2}$, is increasing worldwide [1]. From 2011-2014, approximately $36 \%$ of adults in the United

John J. Veillette

john.veillette@gmail.com

1 Department of Pharmacy Practice, Loma Linda University School of Pharmacy, Loma Linda, CA, USA

2 Department of Pharmaceutical and Administrative Sciences, Loma Linda University School of Pharmacy, Loma Linda, CA, USA

3 JMI Laboratories, North Liberty, IA, USA

4 Department of Pharmacy, Loma Linda University Medical Center, Loma Linda, CA, USA

5 Loma Linda University School of Medicine, Loma Linda, CA, USA
States were obese, which may grow to $40-50 \%$ by 2030 [2, 3]. Limited data exist regarding optimal antibiotic dosing in this population, which is problematic because obesity is associated with increased mortality in the critically ill and may be a predictor of treatment failure [4-8].

Piperacillin-tazobactam (P-T) is commonly prescribed at $3.375 \mathrm{~g}$ or $4.5 \mathrm{~g}$ every $6 \mathrm{~h}$ [9]. However, clinical studies evaluating this dosing either did not specify weight or enrolled mostly non-obese patients [10-13]. One study in patients with complicated intra-abdominal infections found a $21 \%$ lower cure rate for those with BMI $\geq 30 \mathrm{~kg} / \mathrm{m}^{2}$ treated with P-T $3.375 \mathrm{~g}$ every $6 \mathrm{~h}$ [14]. Several studies and case reports have described low P-T concentrations in obese patients, yet higher dosing regimens remain to be evaluated [15-19]. Therefore, the objective of our study was to investigate the pharmacokinetics, pharmacodynamics, and safety of standard- and high-dose P-T in obese patients at our institution. 


\section{Key Points}

The pharmacokinetics, pharmacodynamics, and safety of piperacillin-tazobactam doses up to $9 \mathrm{~g}$ every $6 \mathrm{~h}$ were evaluated in obese adults.

Some patients required high P-T doses for target attainment, but dosing requirements were highly variable.

Doses up to $6.75 \mathrm{~g}$ or $9 \mathrm{~g}$ every $6 \mathrm{~h}$ may be tolerable.

\section{Patients and Methods}

Approval was obtained from Loma Linda University Medical Center's Institutional Review Board (IRB) prior to study commencement (study \#5130259). The trial was registered and maintained on clinicaltrials.gov (NCT01923363).

\subsection{Patients}

Adult patients 18-89 years of age at Loma Linda University Medical Center (LLUMC) who were obese (BMI $\geq 30 \mathrm{~kg} / \mathrm{m}^{2}$ ) and receiving P-T for a suspected or confirmed infection were eligible for enrollment. Dedicated vascular access (central or arterial line) was required for blood draws. Patients with pre-existing seizure or hematologic disorders, hepatic insufficiency (cirrhosis with Child-Pugh class B or above), $\beta$-lactam allergy, or who were pregnant were excluded. Approval was obtained from the primary care team attending physician, and written informed consent was obtained from each patient or legally authorized representative prior to study enrollment. Demographic and laboratory data were collected at baseline. Acute Physiology and Chronic Health Evaluation II (APACHE II) and Sequential Organ Failure Assessment (SOFA) scores were calculated for those in an intensive care unit (ICU). Creatinine clearance $(\mathrm{CrCl})$ was calculated using a lean body weight estimate formula in the Cockcroft-Gault equation based on its performance in morbid obesity [20]. Patients were divided into four groups based on their $\mathrm{CrCl}$ at study enrollment: $>80 \mathrm{~mL} / \mathrm{min}$ (Group I), $40-80 \mathrm{~mL} / \mathrm{min}$ (Group II), $<40 \mathrm{~mL} / \mathrm{min}$ (Group III), and hemodialysis (Group IV).

\subsection{Antibiotic Regimen and Blood Sampling}

Patients were receiving standard P-T dosing (every $6 \mathrm{~h}$ infused over $30 \mathrm{~min}$ per institutional order sets) at the discretion of the primary care team upon enrollment. Following enrollment, blood samples were obtained surrounding the next standard dose: a sample before, then three consecutive samples at 1,3 , and $5 \mathrm{~h}$ after the 30 -min infusion. The following dose was then increased according to the study protocol: up to $6.75 \mathrm{~g}$ ( $6 \mathrm{~g}$ piperacillin and $0.75 \mathrm{~g}$ tazobactam) for patients $105-139 \mathrm{~kg}$, and up to $9 \mathrm{~g}$ ( $8 \mathrm{~g}$ piperacillin and $1 \mathrm{~g}$ tazobactam) for patients $\geq 140 \mathrm{~kg}$ (50\% and $100 \%$ higher than the highest approved dose, $4.5 \mathrm{~g}$, respectively). Lower doses could be chosen at the discretion of the attending physician (e.g., increasing a dose of 2.25 to $4.5 \mathrm{~g}$ ). Doses were capped at $6.75 \mathrm{~g}$ for $\mathrm{CrCl} \leq 10 \mathrm{~mL} / \mathrm{min}$ or hemodialysis patients. After administration of the first high dose, blood samples were collected at 1,3 , and $5 \mathrm{~h}$ after the 30 -min infusion. Patients were then continued on a high-dose regimen (every $6 \mathrm{~h}$ infused over $30 \mathrm{~min}$ ) until the primary team stopped P-T or switched to another antibiotic.

\subsection{Safety}

Each patient was monitored daily for adverse events for 2 weeks after P-T was discontinued or until hospital discharge. Laboratory values were obtained at the discretion of the primary team: serum creatinine, blood urea nitrogen, white blood cell count, hemoglobin, hematocrit, platelets, aspartate aminotransferase, alanine aminotransferase, total bilirubin, and alkaline phosphatase (when available) were followed up for signs of acute abnormalities. Adverse events were promptly reported to the primary team and the IRB. The Naranjo scale was used to determine the likelihood of high-dose P-T having caused the adverse event [21]. The primary team could reduce the P-T dose or discontinue P-T at their discretion.

\subsection{Sample Processing and Storage}

All blood samples were allowed to clot for $10 \mathrm{~min}$ and were centrifuged at $4000 \mathrm{rpm}$ for $10 \mathrm{~min}$ at room temperature. Serum was extracted and stored at $-80^{\circ} \mathrm{C}$ until analyzed. All samples were frozen within 90 min of the blood draw.

\subsection{Assay}

Samples were thawed at room temperature. Penicillin G internal standard was added to the patient sample and loaded onto a HyperSep C8 extraction cartridge (Thermo Scientific, Waltham, MA, USA), which was pre-conditioned with $1 \mathrm{~mL}$ analytical grade methanol, then $1 \mathrm{~mL}$ nanopure water. Cartridges were centrifuged at $1500 \mathrm{rpm}$ for $1 \mathrm{~min}$ at room temperature, and the eluent was collected for analysis by highperformance liquid chromatography (HPLC). Samples were analyzed for P-T concentrations by a single assay using a previously validated HPLC method [22]. The lower limits of quantitation and detection were 1.0 and $0.4 \mathrm{mg} / \mathrm{L}$ for piperacillin and 0.78 and $0.20 \mathrm{mg} / \mathrm{L}$ for tazobactam, respectively. The coefficient of variation for intra- versus inter-day precision ranged from $1.4-3.4 \%$ versus $2.8-4.8 \%$ for piperacillin and from $3.4-4.2 \%$ versus $3.9-4.3 \%$ for tazobactam. Each 
sample was run in duplicate, and an average concentration was calculated from the two runs.

\subsection{Pharmacokinetic Calculations}

Log-linear plots were made in Microsoft Excel. Elimination rate constants $\left(K_{\mathrm{el}}\right)$ were determined from the three P-T concentrations after each standard and high dose using least squares regression. $R^{2}$ values $<0.90$ for the regression line prompted evaluation for erroneous blood samples, defined as: first level drawn in the distribution phase ( $K_{\mathrm{el}}$ for concentrations 1 and 2 different than 2 and 3); concentration lower than expected (e.g., from fluid bolus); or significant interference on the HPLC chromatograph. Concentrations judged to be erroneous were excluded from the analysis. Half-lives were calculated using Eq. 1:

Half-life $=0.693 / K_{\mathrm{el}}$.

Extrapolated peaks and troughs for standard- and highdose P-T were calculated with Eq. 2 using standard- and high-dose $K_{\mathrm{el}}$ values, respectively ( $C_{1}$ is concentration $1, C_{2}$ is concentration 2 , and $t$ is time):

$C_{2}=C_{1} \times e^{-K_{\mathrm{el}} \times t}$

Volumes of distribution $(V \mathrm{~d})$ were calculated using the Sawchuk-Zaske method [23]. Standard versus high-dose $K_{\text {el }}$ and $V \mathrm{~d}$ were compared for each patient using least squares regression to see if any relationship between dose and pharmacokinetic values could be determined.

\subsection{Pharmacodynamic Calculations}

Piperacillin free trough concentrations $>16 \mathrm{mg} / \mathrm{L}$ and tazobactam free trough concentrations $>4 \mathrm{mg} / \mathrm{L}(100 \%$ $f \mathrm{~T}>16 / 4 \mathrm{mg} / \mathrm{L}$ ) were chosen as a pharmacodynamic target based on in vitro and clinical studies [24-35], a worstcase scenario for susceptible Enterobacteriaceae (breakpoint $=16 / 4 \mathrm{mg} / \mathrm{L}$ ) [9], and Gram-negative minimum inhibitory concentration (MIC) ${ }_{90}$ values from the SENTRY Antimicrobial Surveillance Program (Table S1 in Supplemental material). P-T free steady state trough concentrations $\left(C_{\text {min,ss }}\right)$ were calculated using Eq. 3, where $t_{\text {inf }}$ is the infusion time, tau is the dosing interval, and 0.7 is the average unbound fraction for both piperacillin and tazobactam $[9$, 23]:

$C_{\text {min,SS }}=\frac{0.7 \times \operatorname{Dose}\left(1-e^{-K_{\mathrm{el}} \times t_{\text {inf }}}\right) \times e^{-K_{\mathrm{el}}\left(\mathrm{tau}-t_{\mathrm{inf}}\right)}}{t_{\mathrm{inf}} \times K_{\mathrm{el}} \times V \mathrm{~d} \times\left(1-e^{-K_{\mathrm{el}} \times \text { tau }}\right)}$

Various standard- and high-dose P-T regimens administered via standard infusion (30 min), extended infusion $(3 \mathrm{~h})$, and continuous infusion ( $24 \mathrm{~h}$ ) were simulated for each patient using their own pharmacokinetic values to calculate the likelihood of target attainment.

\section{Results}

\subsection{Patients}

A total of 434 obese patients receiving P-T were screened prospectively between June 2013 and January 2016. Of these, 63 met the inclusion criteria, of whom 12 declined study participation, 9 were excluded by the primary care team, and 13 had P-T switched or discontinued prior to enrollment. The remaining 29 unique patients were enrolled. All 29 received at least one high dose of P-T and were included in the safety analysis. One patient had her central line removed after giving informed consent. She was still given high-dose P-T (9 g every $6 \mathrm{~h}$ ) but no blood samples were obtained. This left 28 patients in the final pharmacokinetic/pharmacodynamic analysis. The median duration of standard-dose P-T prior to enrollment was 2 days (range 1-8 days), and the median duration of high dose P-T was 2 days (range 1-12 days). Baseline patient characteristics stratified by $\mathrm{CrCl}$ are shown in Table 1 .

\subsection{Safety}

Of 29 patients, 1 (3\%) experienced an adverse event deemed probably attributable to high-dose P-T. Adverse events in an additional nine patients $(31 \%)$ were deemed possibly attributable due plausible alternative explanations (Table 2). Average high-dose peak and trough concentrations were not significantly different between those who experienced an adverse event and those who did not, although this analysis was limited by the small sample size. Specific trough concentrations for patients experiencing an adverse event are displayed in Table 2 .

\subsection{Pharmacokinetic Analysis}

P-T concentration-time profiles within each Group are displayed in the Supplemental material (Figures S1-S5). P-T half-lives were highly variable, ranging from 0.5 to $10.6 \mathrm{~h}$ for piperacillin and 0.9-15.0 h for tazobactam (Table 3). Average half-life generally increased with decreasing $\mathrm{CrCl}$ across Groups I-IV; however, individual half-lives could not be predicted based on $\mathrm{CrCl}\left(R^{2}=0.62\right.$ for least squares regression). $V \mathrm{~d}$ was also highly variable, ranging from 13.6 to $54.8 \mathrm{~L}$ for piperacillin, and from 11.5 to $60.1 \mathrm{~L}$ for tazobactam. The average $V \mathrm{~d}$ for piperacillin was $24.1 \pm 8.8 \mathrm{~L}$ $(0.19 \pm 0.08 \mathrm{~L} / \mathrm{kg})$, which was larger than the population mean of $18 \mathrm{~L}(0.24 \mathrm{~L} / \mathrm{kg} \times 75 \mathrm{~kg})$ [9]. Average $V \mathrm{~d}$ for tazobactam was $27.0 \pm 10.6 \mathrm{~L}(0.21 \pm 0.09 \mathrm{~L} / \mathrm{kg})$. Individual 
Table 1. Baseline characteristics

\begin{tabular}{|c|c|c|c|c|c|c|c|c|c|}
\hline Group & $n$ & $\begin{array}{l}\text { Creatinine } \\
\text { clearance }(\mathrm{mL} / \\
\text { min) }\end{array}$ & Age (years) & Weight $(\mathrm{kg})$ & $\mathrm{BMI}\left(\mathrm{kg} / \mathrm{m}^{2}\right)$ & $\begin{array}{l}\text { Total bilirubin } \\
(\mathrm{mg} / \mathrm{dL})\end{array}$ & $\begin{array}{l}\text { ICU patients } \\
n(\%)\end{array}$ & $\begin{array}{l}\text { APACHE II } \\
\text { Score }^{\mathrm{a}}\end{array}$ & SOFA Score ${ }^{a}$ \\
\hline I & 12 & $122(82-205)$ & $46(19-68)$ & $127(105-151)$ & $41(32-57)$ & $0.6(0.2-1.5)$ & $6(50.0 \%)$ & $16(9-26)$ & $6(5-7)$ \\
\hline II & 8 & $55(42-72)$ & $57(43-79)$ & $142(118-205)$ & 47 (34-65) & $0.8(0.4-1.9)$ & $7(87.5 \%)$ & $17(4-42)$ & $7(0-14)$ \\
\hline III & 5 & $29(22-36)$ & $60(48-74)$ & $121(109-137)$ & $38(33-50)$ & $1.3(0.3-3.0)$ & $5(100 \%)$ & $23(16-28)$ & $9(6-11)$ \\
\hline IV & 3 & Hemodialysis & $42(29-61)$ & $136(118-156)$ & $49(40-55)$ & $0.5(0.3-0.9)$ & $3(100 \%)$ & $26(21-29)$ & $9(7-11)$ \\
\hline
\end{tabular}

Group definitions by creatinine clearance were: Group I: > $80 \mathrm{~mL} / \mathrm{min}$, Group 2: 40-80 mL/min, Group III: < $40 \mathrm{~mL} / \mathrm{min}$, and Group IV: hemodialysis. Values are expressed as mean (range), or number (percent)

Indications for P-T included: Pneumonia $(n=17)$, Intra-abdominal infection $(n=4)$, Skin and soft tissue infection $(n=3)$, Urinary tract infection $(n=3)$, and Bone and Joint infection $(n=1)$

$B M I$ body mass index, ICU intensive care unit, APACHE II acute physiology and chronic health evaluation II, SOFA sequential organ failure assessment

${ }^{a}$ APACHE II and SOFA scores were only calculated for ICU patients

$V$ d could not be predicted based on weight $\left(R^{2}=0.30\right.$ for least squares regression). Average P-T peak and trough concentrations generally increased by dose across Groups I-IV (Table 3), except for the four patients receiving $9 \mathrm{~g}$ (three in Group I and one in Group II), who had higher peaks but lower troughs than those receiving $6.75 \mathrm{~g}$. This could be explained by their shorter P-T half-lives relative to patients receiving $6.75 \mathrm{~g}$.

No relationship was found between pharmacokinetic values and ICU status, APACHE II score, or SOFA score, but these analyses were limited by the small sample size. Comparing standard- and high-dose pharmacokinetic values for each patient, the half-lives were similar $\left(R^{2}=0.91\right.$ for zero-intercept least squares regression line), but significant variability was seen in $V \mathrm{~d}\left(R^{2}=0.06\right)$. No relationship was observed between increasing P-T dose and half-life or $V \mathrm{~d}$.

\subsection{Pharmacodynamic Analysis}

Each patient's standard-dose pharmacokinetic values were used for target attainment calculations because they were mostly drawn under steady-state conditions (see Supplemental Material) and pharmacokinetic values were not found to be dose-dependent. Predicted versus measured high-dose concentrations were then compared for validation. The highest approved dose, $4.5 \mathrm{~g}$ every $6 \mathrm{~h}$ infused over $30 \mathrm{~min}$, would have met the pharmacodynamic target for only one patient $(8.3 \%)$ in Group I, five $(62.5 \%)$ in Group II, and four $(80 \%)$ in Group III (Table 4$)$. Predicted free steadystate piperacillin troughs on $4.5 \mathrm{~g}$ every $6 \mathrm{~h}$ are displayed in Fig. 1, which shows that Group I patients $(\mathrm{CrCl}>80 \mathrm{~mL} /$ min) were especially prone to low troughs. Indeed, this was reflected in the measured concentrations for patients receiving this dose (and lower doses) upon study enrollment. ICU status did not appear to impact target attainment in Group I
[0/6 (0\%) for non-ICU vs. $1 / 6(17 \%)$ for ICU, $p=1.0]$, but this analysis was limited by the small sample size.

Of the simulated regimens, continuous infusions would have achieved the highest percent target attainment across all groups [25/28 (89\%) for $13.5 \mathrm{~g}, 27 / 28$ (96\%) for $18 \mathrm{~g}$, and 28/28 (100\%) for $27 \mathrm{~g}$ over $24 \mathrm{~h}$ ]. Among the extended infusion regimens, $9 \mathrm{~g}$ every $6 \mathrm{~h}$ would have led to target attainment for the most patients, except for $4 / 12$ patients (33\%) in Group I (Fig. 1). Three of these four patients had calculated $\mathrm{CrCl}$ values suggestive of augmented renal clearance $[(>130 \mathrm{~mL} / \mathrm{min})$, range $149-205 \mathrm{~mL} / \mathrm{min}]$. All four had piperacillin half-lives less than $1 \mathrm{~h}$ (range $0.5-0.8 \mathrm{~h}$ ), and their volumes of distribution ranged from 14.1 to $21.5 \mathrm{~L}$. A continuous infusion would have been needed for target attainment in these patients.

\section{Discussion}

It is important to consider optimal pharmacokinetic/pharmacodynamic targets when dosing P-T. Regarding tazobactam, percent time over a threshold concentration is best associated with P-T efficacy. Identified thresholds have ranged from 0.25 to $2 \mathrm{mg} / \mathrm{L}$, yet re-growth or a minimum killing effect was seen even at $2 \mathrm{mg} / \mathrm{L}$ for some isolates [24-26]. In vitro data also suggest that higher tazobactam concentrations enhance piperacillin activity against Gram-negative isolates [27-29]. Regarding piperacillin, studies evaluating $\beta$-lactams have found that $100 \% f \mathrm{~T}>\mathrm{MIC}$ (not the more traditional $50 \% f \mathrm{~T}>\mathrm{MIC}$ ) is associated with improved outcomes, and that free troughs $\geq 4-5$ times the MIC may optimize efficacy [30-35]. True MICs would often be unknown in clinical practice, but the SENTRY data revealed an $\mathrm{MIC}_{90}$ value of $16 / 4 \mathrm{mg} / \mathrm{L}$ for Enterobacteriaceae isolates, and even higher values for Pseudomonas aeruginosa and Acinetobacter spp. 


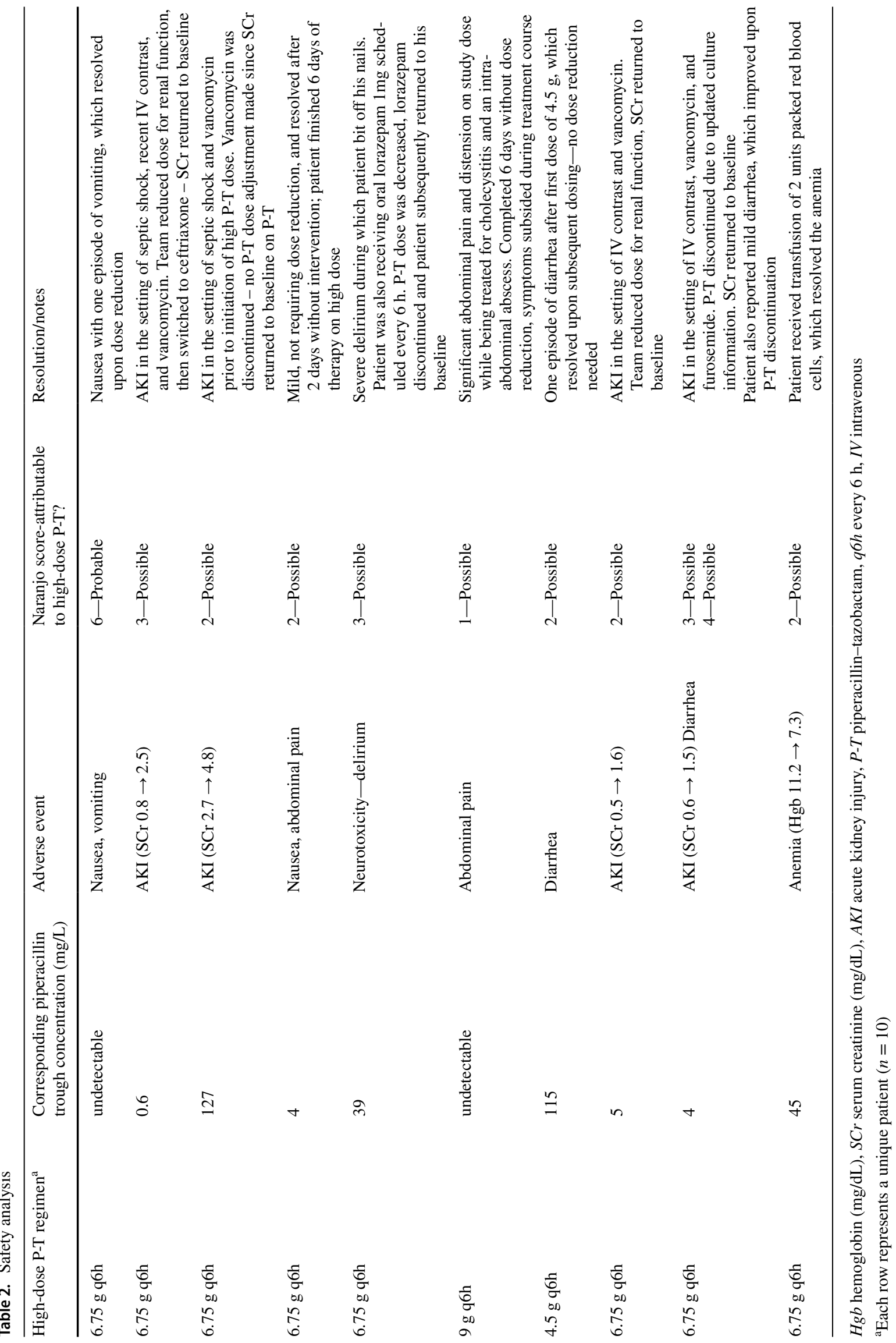


Table 3. Pharmacokinetics of piperacillin and tazobactam

\begin{tabular}{|c|c|c|c|c|c|c|c|c|c|}
\hline \multirow{2}{*}{$\overline{\text { Group }}$} & \multirow[b]{2}{*}{$n$} & \multicolumn{4}{|l|}{ Piperacillin } & \multicolumn{4}{|l|}{ Tazobactam } \\
\hline & & Half-life (h) & $\begin{array}{l}\text { Volume of dis- } \\
\text { tribution (L) }\end{array}$ & $C_{\max }(\mathrm{mg} / \mathrm{L})^{\mathrm{a}}$ & $C_{\min }(\mathrm{mg} / \mathrm{L})^{\mathrm{a}}$ & Half-life (h) & $\begin{array}{l}\text { Volume of dis- } \\
\text { tribution (L) }\end{array}$ & $C_{\max }(\mathrm{mg} / \mathrm{L})^{\mathrm{a}}$ & $C_{\min }(\mathrm{mg} / \mathrm{L})^{\mathrm{a}}$ \\
\hline \multicolumn{10}{|l|}{ I } \\
\hline $3.375 \mathrm{~g}$ & 7 & $1.2(0.5-2.7)$ & $\begin{array}{l}19.0(14.1- \\
24.7)\end{array}$ & $\begin{array}{l}136(107- \\
179)\end{array}$ & $\begin{array}{c}8.9(0.0- \\
44.1)^{\mathrm{b}}\end{array}$ & $2.4(1.1-3.0)$ & $\begin{array}{l}28.0(15.6- \\
34.1)\end{array}$ & $\begin{array}{l}15.8(9.6- \\
28.2)\end{array}$ & $3.7(0.3-8.2)$ \\
\hline $4.5 \mathrm{~g}$ & 5 & $1.5(0.8-2.4)$ & $\begin{array}{l}20.9(13.6- \\
27.6)\end{array}$ & $\begin{array}{l}190(149- \\
247)\end{array}$ & $\begin{array}{c}11.1(1.4- \\
18.2)\end{array}$ & $2.4(2.1-2.8)$ & $\begin{array}{l}27.0(17.1- \\
33.4)\end{array}$ & $\begin{array}{l}21.5(17.7- \\
29.1)\end{array}$ & $4.5(2.9-7.2)$ \\
\hline $6.75 \mathrm{~g}$ & 9 & $1.8(1.1-3.0)$ & $\begin{array}{l}27.7(14.9- \\
61.0)\end{array}$ & $\begin{array}{c}237(96.4- \\
368)\end{array}$ & $\begin{array}{l}27.0 \\
\quad(8.6-72.6)\end{array}$ & $2.6(1.3-5.0)$ & $\begin{array}{l}\text { 33.0 (17.6- } \\
58.7)\end{array}$ & $\begin{array}{l}28.7(16.0- \\
42.9)\end{array}$ & $5.5(1.9-8.5)$ \\
\hline $9 \mathrm{~g}$ & 3 & $0.9(0.6-1.4)$ & $\begin{array}{l}20.1(17.3- \\
22.3)\end{array}$ & $\begin{array}{l}332(283- \\
417)\end{array}$ & $\begin{array}{l}10.3(0.57- \\
29.4)\end{array}$ & $1.6(0.9-2.2)$ & $\begin{array}{l}32.0(25.4- \\
38.5)\end{array}$ & $\begin{array}{l}30.1(21.8- \\
38.5)\end{array}$ & $2.9(0.2-5.5)$ \\
\hline \multicolumn{10}{|l|}{ II } \\
\hline $3.375 \mathrm{~g}$ & 6 & $3.9(1.9-6.8)$ & $\begin{array}{l}31.6(20.4- \\
54.8)\end{array}$ & $\begin{array}{l}144(99.1- \\
184)\end{array}$ & $\begin{array}{l}47.4(24.1- \\
75.4)\end{array}$ & $4.6(1.5-7.7)$ & $\begin{array}{l}33.3(15.3- \\
60.1)\end{array}$ & $\begin{array}{l}20.8(10.4- \\
27.6)\end{array}$ & $8.5(2.1-16.9)$ \\
\hline $4.5 \mathrm{~g}$ & 2 & $2.7(2.6-2.9)$ & $\begin{array}{l}20.3(19.3- \\
21.3)\end{array}$ & $\begin{array}{l}214(211- \\
215)\end{array}$ & $\begin{array}{l}52.5(49.4- \\
55.6)\end{array}$ & $2.8(1.7-4.0)$ & $\begin{array}{l}17.1(11.5- \\
22.7)\end{array}$ & $\begin{array}{l}39.7(28.3- \\
51.1)\end{array}$ & $8.1(5.3-10.9)$ \\
\hline $6.75 \mathrm{~g}$ & 7 & $3.8(2.2-9.4)$ & $\begin{array}{l}31.7(18.3- \\
54.5)\end{array}$ & $\begin{array}{l}244(172- \\
350)\end{array}$ & $\begin{array}{l}72.6(37.5- \\
128)\end{array}$ & $4.8(2.2-13.5)$ & $\begin{array}{l}36.5(16.5- \\
74.0)\end{array}$ & $\begin{array}{l}31.7(19.7- \\
44.2)\end{array}$ & $11.1(5.6-19.0)$ \\
\hline $9 \mathrm{~g}$ & 1 & 2.5 & 48.8 & 191 & 54.1 & - & - & - & - \\
\hline \multicolumn{10}{|l|}{ III } \\
\hline $2.25 \mathrm{~g}$ & 2 & $4.3(2.0-6.5)$ & $\begin{array}{l}22.9(16.9- \\
28.8)\end{array}$ & $\begin{array}{l}176(94.6- \\
258)\end{array}$ & $\begin{array}{l}78.8(14.5- \\
143)\end{array}$ & - & - & - & - \\
\hline $3.375 \mathrm{~g}$ & 1 & 10.7 & 30.5 & 296 & 207 & 11.1 & 21.4 & 46.3 & 32.8 \\
\hline $4.5 \mathrm{~g}$ & $5^{c}$ & $6.2(2.3-10.6)$ & $\begin{array}{l}26.3(20.0- \\
32.9)\end{array}$ & $\begin{array}{l}265(163- \\
327)\end{array}$ & $\begin{array}{l}142(31.1- \\
223)\end{array}$ & $8.6(4.8-15.0)$ & $\begin{array}{l}23.2(15.5- \\
30.9)\end{array}$ & $\begin{array}{l}45.9(38.8- \\
51.2)\end{array}$ & $\begin{array}{l}26.9(20.8- \\
37.0)\end{array}$ \\
\hline $6.75 \mathrm{~g}$ & 2 & $5.5(5.1-5.8)$ & $\begin{array}{l}29.9(23.5- \\
36.3)\end{array}$ & $\begin{array}{l}299(287- \\
312)\end{array}$ & $148(135-162)$ & $7.2(6.7-7.7)$ & $\begin{array}{l}36.2(27.3- \\
45.2)\end{array}$ & $\begin{array}{l}39.7(38.0- \\
41.4)\end{array}$ & $\begin{array}{l}23.2(23.1- \\
23.3)\end{array}$ \\
\hline \multicolumn{10}{|l|}{$I^{d}$} \\
\hline $2.25 \mathrm{~g}^{\mathrm{e}}$ & 2 & 9.8 & 34.9 & $\begin{array}{c}158(142- \\
173)\end{array}$ & $99(79.6-118)$ & - & - & - & - \\
\hline $3.375 \mathrm{~g}$ & 1 & 8.6 & 28.9 & 223 & 143 & 14.3 & 25.3 & 35.3 & 27.1 \\
\hline $4.5 \mathrm{~g}$ & 1 & 9.1 & 24.6 & 275 & 181 & - & - & - & - \\
\hline $6.75 \mathrm{~g}$ & 2 & $7.4(6.5-8.4)$ & $\begin{array}{l}34.0(29.7- \\
38.3)\end{array}$ & $\begin{array}{c}397(304- \\
490)\end{array}$ & $234(193-274)$ & $5.3(3.1-7.6)$ & $\begin{array}{l}20.8(12.1- \\
29.5)\end{array}$ & $\begin{array}{l}72.1(52.7- \\
91.6)\end{array}$ & $\begin{array}{l}29.5(27.1- \\
31.8)\end{array}$ \\
\hline
\end{tabular}

Rows in roman type represents standard dose upon study enrollment for all patients within the Group. Rows in bold face represents high dose for the same Group of patients. All doses were infused over 30 min every $6 \mathrm{~h}$ unless otherwise noted

Group definitions by creatinine clearance were: Group I: $>80 \mathrm{~mL} / \mathrm{min}$, Group 2: 40-80 mL/min, Group III: < $40 \mathrm{~mL} / \mathrm{min}$, and Group IV: hemodialysis. All values are expressed as mean (range)

$C_{\max }$ maximum concentration (peak), $C_{\min }$ minimum concentration (trough)

${ }^{a}$ Values represent total drug. High dose $\mathrm{C}_{\max }$ and $\mathrm{C}_{\min }$ do not necessarily represent steady state conditions

${ }^{\mathrm{b}}$ Trough concentrations were undetectable for two patients

${ }^{c} n=5$ represents a combination of low and high dose -2 patients were receiving $4.0 \mathrm{~g}$ upon enrollment and were increased to $6.75 \mathrm{~g}$, while 3 patients were receiving either $2.25 \mathrm{~g}$ or $3.375 \mathrm{~g}$ and increased to $4.5 \mathrm{~g}$

${ }^{\mathrm{d}}$ Pharmacokinetic values were calculated between HD sessions. Pre- and post-HD concentrations and intra-HD half-life are not displayed here ${ }^{\mathrm{e}} 1$ patient was receiving $2.25 \mathrm{~g}$ every $12 \mathrm{~h}$ upon enrollment, but half-life and $V \mathrm{~d}$ were unable to be calculated because the blood samples were drawn surrounding hemodialysis. Values were still displayed for $C_{\max }$ and $C_{\min }$

Therefore, we chose $100 \% f \mathrm{~T}>16 / 4 \mathrm{mg} / \mathrm{L}$ as a worst-case scenario pharmacodynamic target for susceptible Enterobacteriaceae, recognizing that true MICs are often lower and would have required lower doses than those calculated for target attainment.
Achieving optimal P-T concentrations in obese patients is challenging, and studies have had conflicting recommendations for best dosing practices. Alobaid et al. suggested weight-based dosing in one study [19] and more frequent dosing ( 4 g every $4 \mathrm{~h}$ ) or prolonged infusions in another 
Table 4. Predicted piperacillin-tazobactam pharmacodynamic target attainment

\begin{tabular}{|c|c|c|c|c|c|c|c|c|c|c|c|}
\hline \multirow[t]{2}{*}{ Dosage regimen } & \multicolumn{5}{|c|}{ Standard infusion over $30 \mathrm{~min}$} & \multicolumn{3}{|c|}{ Extended infusion over $3 \mathrm{~h}$} & \multicolumn{3}{|c|}{$\begin{array}{l}\text { Continuous infusion over } \\
24 \mathrm{~h}\end{array}$} \\
\hline & $2.25 \mathrm{~g} \mathrm{q} 6 \mathrm{~h}$ & $3.375 \mathrm{~g} \mathrm{q} 6 \mathrm{~h}$ & $4.5 \mathrm{~g} \mathrm{q} 6 \mathrm{~h}$ & $6.75 \mathrm{~g} \mathrm{q} 6 \mathrm{~h}$ & $9 \mathrm{~g} \mathrm{q} 6 \mathrm{~h}$ & $4.5 \mathrm{~g} \mathrm{q} 6 \mathrm{~h}$ & $6.75 \mathrm{~g} \mathrm{q} 6 \mathrm{~h}$ & $9 \mathrm{~g} \mathrm{q6h}$ & $13.5 \mathrm{~g}$ & $18 \mathrm{~g}$ & $27 \mathrm{~g}$ \\
\hline Group I $(n=12)$ & $1(8.3)$ & $1(8.3)$ & $1(8.3)$ & $2(16.7)$ & $4(33.3)$ & $3(25)$ & $5(41.7)$ & $8(66.7)$ & $9(75)$ & $11(92)$ & $12(100)$ \\
\hline Group II $(n=8)$ & $4(50)$ & $5(62.5)$ & $5(62.5)$ & $7(87.5)$ & $7(87.5)$ & $7(87.5)$ & $8(\mathbf{1 0 0})$ & $8(100)$ & $8(100)$ & $8(100)$ & $8(\mathbf{1 0 0})$ \\
\hline Group III $(n=5)$ & $4(80)$ & $4(80)$ & $4(80)^{\mathrm{a}}$ & $5(100)$ & $5(100)$ & $5(100)$ & $5(100)$ & $5(100)$ & $5(100)$ & $5(100)$ & $5(100)$ \\
\hline Group IV $(n=3)$ & $3(100)$ & $3(100)$ & $3(100)$ & $3(100)$ & $3(100)$ & $3(100)$ & $3(\mathbf{1 0 0})$ & $3(100)$ & $3(100)$ & $3(\mathbf{1 0 0})$ & $3(100)$ \\
\hline
\end{tabular}

Values represent number (percent) of patients in each group meeting the target (100\% $f \mathrm{~T}>16 / 4 \mathrm{mg} / \mathrm{L})$ at each theoretical dosing regimen using each patient's own $\mathrm{K}_{\mathrm{el}}$ and $\mathrm{V}$. Bolded values represent $100 \%$ target attainment for a given dosage regimen. Patients for whom tazobactam data were missing were assumed to have met the tazobactam pharmacodynamic target and were evaluated based on piperacillin concentrations only. Group definitions by creatinine clearance were: Group I: $>80 \mathrm{~mL} / \mathrm{min}$, Group 2: 40-80 mL/min, Group III: < $40 \mathrm{~mL} / \mathrm{min}$, and Group IV: hemodialysis

$\mathrm{CrCl}$ creatinine clearance, $f T$ free trough concentration, $K_{e l}$ elimination rate constant, $V d$ volume of distribution, $q 6 h$ every $6 \mathrm{~h}$

${ }^{a}$ The one Group III patient not meeting the target on $4.5 \mathrm{~g}$ q6h had a short piperacillin half-life of $2.0 \mathrm{~h}$ (despite an estimated $\mathrm{CrCl}$ of $27 \mathrm{~mL} /$ $\min$ ), and a large $V \mathrm{~d}$ of $28.8 \mathrm{~L}$

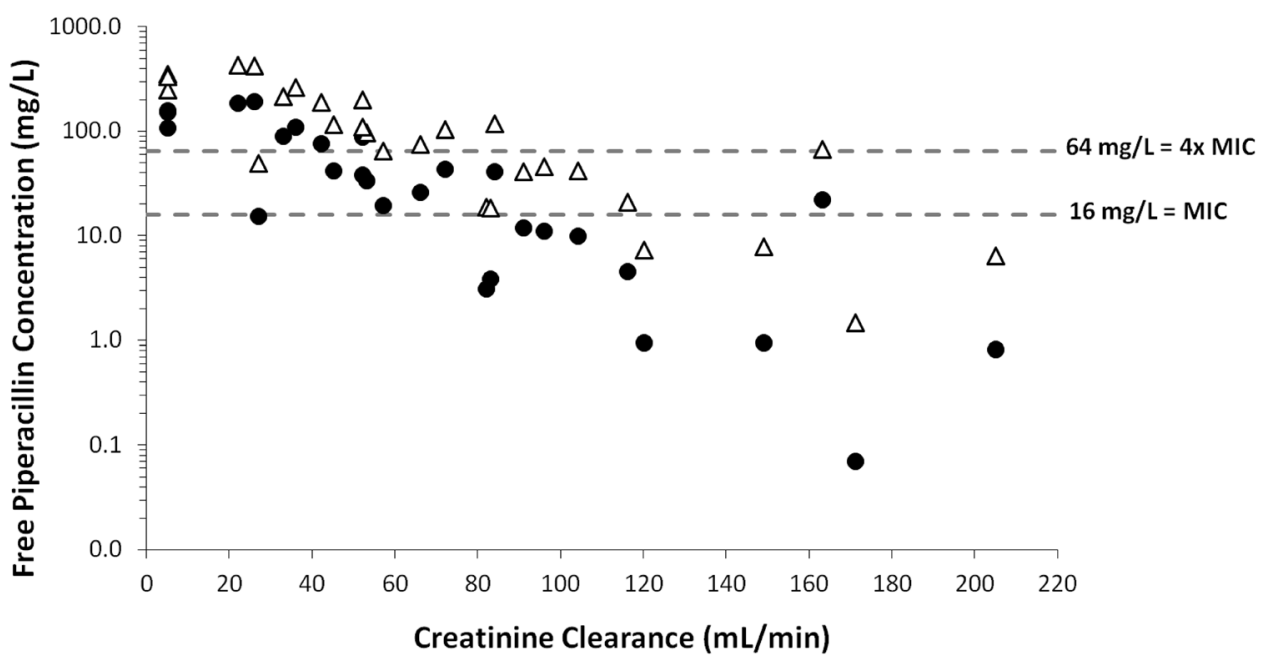

Fig. 1 Predicted free piperacillin steady-state trough concentrations for the same patients on two different dosing regimens: Piperacillintazobactam (P-T) $4.5 \mathrm{~g}$ every $6 \mathrm{~h}$ infused over $30 \mathrm{~min}$ (filled circle), and P-T 9 g every $6 \mathrm{~h}$ infused over $3 \mathrm{~h}$ (open triangle). Creatinine clearance $(\mathrm{CrCl})$ was calculated via the Cockcroft-Gault equation using a lean body weight estimate formula. The three hemodialysis patients were assigned an arbitrary $\mathrm{CrCl}$ of $5 \mathrm{~mL} / \mathrm{min}$ due to inability to calculate an accurate $\mathrm{CrCl}$. MIC minimum inhibitory concentration
[36] based on low piperacillin concentrations in critically ill obese patients. Hites et al. suggested higher P-T dosing regimens based on concentrations in 31 non-critically ill obese patients [16]. All three studies identified $\mathrm{CrCl}$ as a key predictor of failure to meet pharmacodynamic targets. This was similar to our data, which showed that patients with $\mathrm{CrCl}>80 \mathrm{~mL} / \mathrm{min}$ (and particularly those with $\mathrm{CrCl}$ $>120-130 \mathrm{~mL} / \mathrm{min}$ ) were prone to subtherapeutic concentrations. Authors of multiple studies have also concluded that P-T therapeutic drug monitoring (TDM) should be routinely performed in critically ill obese patients based on the significant pharmacokinetic variability observed $[36,37]$. We observed similar variability with a wide range of pharmacokinetic values that could not be predicted by patient characteristics. Conversely, two studies concluded that standard P-T doses achieved adequate concentrations in obese patients [38, 39]; however, both studies chose lower targets of 50\% $f \mathrm{~T}>\mathrm{MIC}$, which may be suboptimal.

Standard P-T doses were sufficient for some obese patients in our study. However, higher doses were needed for others, which raises the question of safety. Of the five patients receiving $9 \mathrm{~g}$ every $6 \mathrm{~h}$, only one experienced an adverse event, which did not require a dose reduction. We captured all adverse events (regardless of cause) for transparency, but in nine of ten cases it was unclear if high-dose P-T was responsible for the adverse event due to plausible alternative explanations. Four of the ten experienced AKI while on concomitant vancomycin [40], but they had 
numerous other risk factors, including IV contrast, diuretics, vasopressors, and septic shock. Our data suggest that high-dose P-T with appropriate clinical monitoring may be feasible if needed to overcome low concentrations in obese patients. Administration of standard-dose P-T via extended or continuous infusion may also improve target attainment in these patients based on our pharmacodynamic analysis.

Our study had some notable limitations. First, high-dose P-T was often switched or discontinued after a short time. This necessitated drawing blood samples after the first high dose (instead of waiting until steady state). It also made an impact on clinical outcomes less likely; thus, outcomes associated with high-dose P-T were not assessed. Second, the short duration of high-dose therapy may have prevented us from finding adverse drug events. Third, the small number of patients was subject to selection bias and prevented us from performing multivariate analysis to identify predictors of pharmacokinetic values, pharmacodynamic target attainment, or adverse events. Larger prospective studies powered for clinical and safety endpoints are needed to optimize dosing recommendations in obese patients. Fourth, the P-T concentrations obtained from each patient only reflect a brief picture of a population whose pharmacokinetic profiles are highly variable. We also calculated unbound P-T concentrations based on population averages, when plasma protein binding is known to be variable [41]. Direct measurement of unbound concentrations may allow for better individualization of dosing. Fifth, we lacked access to modeling software to evaluate pharmacokinetic parameters using compartmental versus noncompartmental methods or linear versus nonlinear models. While we consider our data to be robust, such modeling may have strengthened our findings. Finally, we did not perform predictive population pharmacokinetic modeling or use concentrations to make real-time adjustments in dosing; however, given the large degree of inter-patient variability, an intervention such as real-time TDM could be valuable.

\section{Conclusions}

Our data suggest that some obese patients may need higher P-T doses to meet pharmacokinetic/pharmacodynamic targets; however, a wide range in dose requirements was observed, which was not predictable from baseline characteristics alone. We found that doses up to $9 \mathrm{~g}$ every $6 \mathrm{~h}$ may be tolerable with close monitoring, although the utility of such aggressive dosing (compared to extended or continuous infusions of standard doses) remains unknown. Prospective randomized studies are needed to determine if interventions such as higher P-T doses, prolonged infusions, or real-time TDM lead to improved outcomes in obese patients, especially in those with rapid elimination and infections due to less-susceptible organisms.
Supplementary Information The online version contains supplementary material available at https://doi.org/10.1007/ s13318-021-00677-1.

Acknowledgements We would like to thank the LLUMC nursing staff for their consideration and coordination of the blood draws, the attending physicians for their cooperation in patient enrollment, and the LLU School of Pharmacy for use of their laboratory facilities and for funding the research.

\section{Declarations}

Funding This work was supported by the Loma Linda University School of Pharmacy [Grant number 360031].

Conflict of interest The authors have no conflicts of interest or financial involvements to disclose.

Ethics approval Approval was obtained from Loma Linda University Medical Center's Institutional Review Board (IRB) prior to study commencement - study \#5130259.

Consent to participate Written informed consent was obtained from each patient or legally authorized representative prior to study enrollment.

Consent for publication Not applicable.

Availability of data and material Upon request from the corresponding author.

Code availability Not applicable.

Author contributions JJV-conceptualization; data curation; formal analysis; investigation; methodology; project administration; resources; software; supervision; validation; visualization; roles/Writing —original draft; Writing — review and editing. SAW — conceptualization; data curation; formal analysis; investigation; methodology; project administration; resources; software; supervision; validation; visualization; writing - review and editing. VKM - conceptualization; data curation; formal analysis; funding acquisition; investigation; methodology; project administration; resources; software; supervision; validation; visualization; writing-review and editing. JT—data curation; formal analysis; investigation; methodology; resources; supervision; validation; visualization; writing - review and editing. RNJ-data curation, formal analysis; investigation; Methodology; resources; supervision; validation; visualization; writing — review and editing. SCF-conceptualization; data curation; formal analysis; investigation; methodology; project administration; resources; software; supervision; validation; visualization; writing - review and editing.

Open Access This article is licensed under a Creative Commons Attribution-NonCommercial 4.0 International License, which permits any non-commercial use, sharing, adaptation, distribution and reproduction in any medium or format, as long as you give appropriate credit to the original author(s) and the source, provide a link to the Creative Commons licence, and indicate if changes were made. The images or other third party material in this article are included in the article's Creative Commons licence, unless indicated otherwise in a credit line to the material. If material is not included in the article's Creative Commons licence and your intended use is not permitted by statutory regulation or exceeds the permitted use, you will need to obtain permission directly from the copyright holder. To view a copy of this licence, visit http://creativecommons.org/licenses/by-nc/4.0/. 


\section{References}

1. World Health Organization. Obesity and overweight. Geneva, Switzerland: WHO; 2016. Fact sheet No. 311. http://www.who. int/mediacentre/factsheets/fs311/en/. Accessed 11 Feb 2017.

2. Ogden CL, Carroll MD, Fryar CD, Flegal KM. Prevalence of obesity among adults and youth: United States, 2011-2014. NCHS Data Brief. 2015;2015(219):1-8.

3. Finkelstein EA, Khavjou OA, Thompson H, et al. Obesity and severe obesity forecasts through 2030. Am J Prev Med. 2012;42(6):563-70.

4. Nasraway SA Jr, Albert M, Donnelly AM, Ruthazer R, Shikora SA, Saltzman E. Morbid obesity is an independent determinant of death among surgical critically ill patients. Crit Care Med. 2006;34(4):964-70.

5. Bercault N, Boulain T, Kuteifan K, Wolf M, Runge I, Fleury JC. Obesity-related excess mortality rate in an adult intensive care unit: a risk-adjusted matched cohort study. Crit Care Med. 2004;32(4):998-1003.

6. Conway EL, Sellick JA, Kurtzhalts K, Mergenhagen KA. Obesity and heart failure: predictors of failure in outpatient skin and soft tissue infections. Antimicrob Agents Chemother. 2017;61(3):e02389-e2416.

7. Longo C, Bartlett G, Macgibbon B, et al. The effect of obesity on antibiotic treatment failure: a historical cohort study. Pharmacoepidemiol Drug Saf. 2013;22(9):970-6.

8. Jeffres MN, Barclay SM, Daly MW, Decerbo MC, Golenia P. Impact of body size descriptors on initial treatment response and thirty-day mortality in patients with gram-negative infections. J Appl Res Clin Exp Ther. 2012;12:30-7.

9. Product Information. ZOSYN® Piperacillin and Tazobactam for Injection, USP. Philadelphia : Wyeth Pharmaceuticals Inc; 2012. (Revised May 2012. [Accessed February 11, 2017]).

10. Joshi M, Metzler M, McCarthy M, Olvey S, Kassira W, Cooper A. Comparison of piperacillin/tazobactam and imipenem/ cilastatin, both in combination with tobramycin, administered every $6 \mathrm{~h}$ for treatment of nosocomial pneumonia. Respir Med. 2006;100(9):1554-65.

11. Brismar B, Malmborg AS, Tunevall G, et al. Piperacillin-tazobactam versus imipenem-cilastatin for treatment of intra-abdominal infections. Antimicrob Agents Chemother. 1992;36(12):2766-73.

12. Tan JS, Wishnow RM, Talan DA, Duncanson FP, Norden CW. Treatment of hospitalized patients with complicated skin and skin structure infections: double-blind, randomized, multicenter study of piperacillin-tazobactam versus ticarcillin-clavulanate. The Piperacillin/Tazobactam Skin and Skin Structure Study Group. Antimicrob Agents Chemother. 1993;37(8):1580-6.

13. Polk HC Jr, Fink MP, Laverdiere M, et al. Prospective randomized study of piperacillin/tazobactam therapy of surgically treated intra-abdominal infection. The Piperacillin/Tazobactam Intra-Abdominal Infection Study Group. Am Surg. 1993;59(9):598-605.

14. Zakrison TL, Hille DA, Namias N. Effect of body mass index on treatment of complicated intra-abdominal infections in hospitalized adults: comparison of ertapenem with piperacillin-tazobactam. Surg Infect (Larchmt). 2012;13(1):38-42.

15. Cheatham SC, Fleming MR, Healy DP, et al. Steady-state pharmacokinetics and pharmacodynamics of piperacillin and tazobactam administered by prolonged infusion in obese patients. Int J Antimicrob Agents. 2013;41(1):52-6.

16. Hites $\mathrm{M}$, Taccone FS, Wolff $\mathrm{F}$, et al. Broad-spectrum $\beta$-lactams in obese non-critically ill patients. Nutr Diabetes. 2014;4:e119.

17. Deman H, Verhaegen J, Willems L, Spriet I. Dosing of piperacillin/tazobactam in a morbidly obese patient. J Antimicrob Chemother. 2012;67(3):782-3.
18. Newman D, Scheetz MH, Adeyemi OA, et al. Serum piperacillin/ tazobactam pharmacokinetics in a morbidly obese individual. Ann Pharmacother. 2007;41(10):1734-9.

19. Alobaid AS, Brinkmann A, Frey OR, et al. What is the effect of obesity on piperacillin and meropenem trough concentrations in critically ill patients? J Antimicrob Chemother. 2016;71(3):696-702.

20. Demirovic JA, Pai AB, Pai MP. Estimation of creatinine clearance in morbidly obese patients. Am J Health Syst Pharm. 2009;66(7):642-8.

21. Naranjo CA, Busto U, Sellers EM, et al. A method for estimating the probability of adverse drug reactions. Clin Pharmacol Ther. 1981;30(2):239-45.

22. Veillette JJ, Winans SA, Forland SC, Maskiewicz VK. A simple and rapid RP-HPLC method for the simultaneous determination of piperacillin and tazobactam in human plasma. J Pharm Biomed Anal. 2016;131:80-6.

23. Sawchuk RJ, Zaske DE. Pharmacokinetics of dosing regimens which utilize multiple intravenous infusions: gentamicin in burn patients. J Pharmacokinet Biopharm. 1976;4:183-95.

24. Nicasio AM, VanScoy BD, Mendes RE, et al. Pharmacokineticspharmacodynamics of tazobactam in combination with piperacillin in an in vitro infection model. Antimicrob Agents Chemother. 2016;60(4):2075-80.

25. Rodriguez CA, Agudelo M, Zuluaga AF, Vesga O. In vivo pharmacodynamics of piperacillin/tazobactam: implications for antimicrobial efficacy and resistance suppression with innovator and generic products. Int J Antimicrob Agents. 2017;49(2):189-97.

26. Lister PD, Prevan AM, Sanders CC. Importance of beta-lactamase inhibitor pharmacokinetics in the pharmacodynamics of inhibitor-drug combinations: studies with piperacillin-tazobactam and piperacillin-sulbactam. Antimicrob Agents Chemother. 1997;41(4):721-7.

27. Kuck NA, Jacobus NV, Petersen PJ, Weiss WJ, Testa RT. Comparative in vitro and in vivo activities of piperacillin combined with the beta-lactamase inhibitors tazobactam, clavulanic acid, and sulbactam. Antimicrob Agents Chemother. 1989;33(11):1964-9.

28. Kuck NA, Petersen PJ, Weiss WJ, Testa RT. In vitro and in vivo efficacy of YTR-830H and piperacillin combinations versus betalactamase-producing bacteria. J Chemother. 1989;1(3):155-61.

29. Dalla Costa T, Nolting A, Rand K, Derendorf H. Pharmacokinetic-pharmacodynamic modelling of the in vitro antiinfective effect of piperacillin-tazobactam combinations. Int J Clin Pharmacol Ther. 1997;35(10):426-33.

30. Roberts JA, Paul SK, Akova M, et al. DALI: defining antibiotic levels in intensive care unit patients: are current $\beta$-lactam antibiotic doses sufficient for critically ill patients? Clin Infect Dis. 2014;58(8):1072-83.

31. Tam VH, McKinnon PS, Akins RL, Rybak MJ, Drusano GL. Pharmacodynamics of cefepime in patients with Gram-negative infections. J Antimicrob Chemother. 2002;50(3):425-8.

32. Aitken SL, Altshuler J, Guervil DJ, et al. Cefepime free minimum concentration to minimum inhibitory concentration (fCmin/MIC) ratio predicts clinical failure in patients with Gram-negative bacterial pneumonia. Int J Antimicrob Agents. 2015;45(5):541-4.

33. Li C, Du X, Kuti JL, Nicolau DP. Clinical pharmacodynamics of meropenem in patients with lower respiratory tract infections. Antimicrob Agents Chemother. 2007;51(5):1725-30.

34. McKinnon PS, Paladino JA, Schentag JJ. Evaluation of area under the inhibitory curve (AUIC) and time above the minimum inhibitory concentration (T $>$ MIC) as predictors of outcome for cefepime and ceftazidime in serious bacterial infections. Int $\mathbf{J}$ Antimicrob Agents. 2008;31(4):345-51.

35. Felton TW, Goodwin J, O'Connor L, et al. Impact of bolus dosing versus continuous infusion of piperacillin and tazobactam on the 
development of antimicrobial resistance in Pseudomonas aeruginosa. Antimicrob Agents Chemother. 2013;57(12):5811-9.

36. Alobaid AS, Wallis SC, Jarrett P, et al. Population pharmacokinetics of piperacillin in nonobese, obese, and morbidly obese critically ill patients. Antimicrob Agents Chemother. 2017;61(3):e01276-e1316.

37. Hites M, Taccone FS, Wolff F, et al. Case-control study of drug monitoring of $\beta$-lactams in obese critically ill patients. Antimicrob Agents Chemother. 2013;57(2):708-15.

38. Sturm AW, Allen N, Rafferty KD, et al. Pharmacokinetic analysis of piperacillin administered with tazobactam in critically ill, morbidly obese surgical patients. Pharmacotherapy. 2014;34(1):28-35.
39. Chung EK, Cheatham SC, Fleming MR, Healy DP, Shea KM, Kays MB. Population pharmacokinetics and pharmacodynamics of piperacillin and tazobactam administered by prolonged infusion in obese and nonobese patients. J Clin Pharmacol. 2015;55(8):899-908.

40. Navalkele B, Pogue JM, Karino S, et al. Risk of acute kidney injury in patients on concomitant vancomycin and piperacillintazobactam compared to those on vancomycin and cefepime. Clin Infect Dis. 2017;64(2):116-23.

41. Wong G, Briscoe S, Adnan S, et al. Protein binding of $\beta$-lactam antibiotics in critically ill patients: can we successfully predict unbound concentrations? Antimicrob Agents Chemother. 2013;57(12):6165-70. 